\title{
ALGEBRAS REALIZED BY $n$ RATIONAL HOMOTOPY TYPES
}

\author{
GREGORY LUPTON
}

(Communicated by Frederick R. Cohen)

\begin{abstract}
I construct an example of a graded algebra that is realized as the rational cohomology algebra of exactly $n$ rational homotopy types, for each natural number $n$. The algebras constructed have trivial multiplicative structure. Similar examples are given of graded Lie algebras realized as the rational homotopy Lie algebra of exactly $n$ rational homotopy types.
\end{abstract}

\section{INTRODUCTION}

A standard procedure in algebraic topology is to associate algebraic objects to a topological space. In rational homotopy theory, for instance, algebraic objects commonly associated to a space $X$ are the rational cohomology algebra $H^{*}(X ; \mathbb{Q})$ and the rational homotopy Lie algebra $\pi_{*}(\Omega X) \otimes \mathbb{Q}$. Given such a procedure, it is natural to consider to what degree nonequivalent spaces can have the same algebraic object associated to them. Now $H^{*}(X ; \mathbb{Q})$ and $\pi_{*}(\Omega X) \otimes \mathbb{Q}$ are invariants not just under homotopy equivalence but under the weaker equivalence relation of rational homotopy equivalence. Thus, two basic questions of rational homotopy theory are as follows: Can exactly $n$ rational homotopy types share the same rational cohomology algebra or the same rational homotopy Lie algebra for any nonnegative integer $n$ ? This paper answers these questions positively by constructing explicit examples.

A space $X$ is said to realize an algebra $H$ or a Lie algebra $L$ if $H^{*}(X ; \mathbb{Q}) \cong$ $H$ or $\pi_{*}(\Omega X) \otimes \mathbb{Q} \cong L$ respectively. A number of authors have computed how many rational homotopy types realize an algebra $H$ for certain specific algebras. All the known examples, however, are either realized by $2^{n}$ rational homotopy types for some $n \geq 0$, or by infinitely many rational homotopy types. Thus the examples of this paper fill a gap in the literature; but are also interesting because they are particularly simple. All algebras and Lie algebras constructed here have trivial multiplicative or bracket structure, i.e., they are the rational cohomology algebras of wedges of spheres, or the rational homotopy Lie algebras of products of Eilenberg-Mac Lane spaces. That there exist algebras or Lie algebras realized

Received by the editors May 8, 1990 .

1980 Mathematics Subject Classification (1985 Revision). Primary 55P62, 55P15.

Key words and phrases. Rational homotopy types, classification. 
by $n$ rational homotopy types is not unexpected; more surprising, perhaps, is the fact that examples exist with such simple structure.

\section{The EXAMPLES}

Before proving the main results, some notation and terminology is established. In general, the standard notation of rational homotopy theory is adopted (see, e.g., [BL] or [NM]). In particular if $W$ is a graded vector space, then $s^{-1} \widetilde{W}$ denotes the desuspension of the corresponding reduced graded vector space. For a reduced graded vector space $V, \mathbb{L}(V)$ denotes the free graded Lie algebra on $V$. The Quillen model of an algebra $H$ is a differential graded Lie algebra, which as a Lie algebra, is the free Lie algebra $\left(\mathbb{L}\left(s^{-1} \widetilde{H}\right)\right.$, and which has a quadratic, degree -1 differential $d$ constructed from the multiplicative structure of $H$ in a standard way [BL, 3.2, QU, Appendix B 6.3]. If $H$ has trivial multiplicative structure, then the differential $d$ is zero. A perturbation of the Quillen model of $H$ is a degree -1 derivation $P$ on $\mathbb{L}\left(s^{-1} \widetilde{H}\right)$ such that $P$ extends bracket length by at least 2 and in addition $(d+P)^{2}=0$ [NM, SS]. Any linear transformation $V \rightarrow \mathbb{L}(V)$ can be extended to a derivation on the free graded Lie algebra $\mathbb{L}(V)$. In particular, suppose $V$ has basis $\left\{\alpha_{1}, \alpha_{2}, \ldots\right\}$ and let $\beta$ be an element in $\mathbb{L}(V)$. Then $\beta \cdot \partial \alpha_{k}$ denotes the derivation of $\mathbb{L}(V)$ that maps $\alpha_{k}$ to $\beta$, and maps $\alpha_{l}$ to zero for $l \neq k$. The basic result concerning perturbations and rational homotopy theory is that any rational homotopy type with rational cohomology isomorphic to $H$ can be represented as $\mathbb{L}\left(s^{-1} \widetilde{H}, d+P\right)$ for some perturbation $P$ [BL, 3.2; NM, 2.4].

According to Schlessinger and Stasheff [SS], there exists a classifying set for each algebra $H$ or Lie algebra $L$, and the cardinality of this set equals that of the set of rational homotopy types that realize $H$ or $L$. The classifying set associated to an algebra $H$ is written as a double quotient

$$
\frac{V_{H}}{\exp \left(\operatorname{ad} \mathscr{L}_{H}^{0}\right)} / \text { Aut } H \text {. }
$$

Here $V_{H}$ is a variety that describes all possible perturbations; $\exp \left(\operatorname{ad} \mathscr{L}_{H}^{0}\right)$ is a group that acts on the variety to give an equivalence relation, and $V_{H} /$ $\exp \left(\operatorname{ad} \mathscr{L}_{H}^{0}\right)$ is the corresponding set of equivalence classes. The variety and the group actions are both constructed directly from the Quillen model of $H$. Finally, Aut $H$ is the group of algebra automorphisms, which induces a group action and hence an equivalence relation on $V_{H} / \exp \left(\operatorname{ad} \mathscr{L}_{H}^{0}\right)$, giving the set of equivalence classes $(*)$. A typical equivalence class in the quotient $V_{H} / \exp \left(\operatorname{ad} \mathscr{L}_{H}^{0}\right)$ is written $\{P\}$, where $P$ is a perturbation. If $P$ and $Q$ belong to the same equivalence class in $V_{H} / \exp \left(\operatorname{ad} \mathscr{L}_{H}^{0}\right)$, then we write $\{P\}=\{Q\}$ or $P \sim_{\exp } Q$. If $\{P\}$ and $\{Q\}$ are two classes in $V_{H} / \exp \left(\operatorname{ad} \mathscr{L}_{H}^{0}\right)$ that belong to the same class in $(*)$, then we write $\{P\} \sim_{\text {Aut }}\{Q\}$. For details of this classification, including a description of the various group actions, see [SS] or [LU]. 
Theorem 1. Let $H=H^{*}\left(S^{5} \vee S^{4 n+4} \vee X ; \mathbb{Q}\right)$, where $X$ is the wedge of $n$ spheres $X=S^{4 n+13} \vee S^{4 n+17} \vee \cdots \vee S^{8 n+9}$. Then $H$ is realized by exactly $n+1$ distinct rational homotopy types.

Proof. It is shown that the set $(*)$, for the given $H$, has $n+1$ elements in it. The Quillen model of $H$, i.e., $\mathbb{L}\left(s^{-1} \widetilde{H} ; d\right)$, has a Lie algebra basis $\left\{x, y, \alpha_{1}, \ldots, \alpha_{n}\right\}$ with degrees described by the following

$$
\begin{aligned}
& \begin{array}{ccc}
s^{-1} \widetilde{H}= & \begin{array}{c}
8 n+8 \\
8 n+4
\end{array} \quad \alpha_{n-1}
\end{array} \\
& \begin{array}{cc}
\vdots & \vdots \\
4 n+8+4 k & \alpha_{k} \\
\vdots & \vdots \\
4 n+16 & \alpha_{2} \\
4 n+12 & \alpha_{1}
\end{array} \\
& 4 n+3 \quad y
\end{aligned}
$$

$4 x$.

Let $P$ be any perturbation. Notice the elements $\left\{y, \alpha_{1}, \ldots, \alpha_{n}\right\}$ are in a range of degrees that is approximately stable. In particular, any bracket of length at least 3 in $\mathbb{L}\left(s^{-1} \widetilde{H}\right)$ which contains at least two entries from $\left\{y, \alpha_{1}, \ldots, \alpha_{n}\right\}$, must be of degree at least $8 n+10$. Thus $P\left(\alpha_{k}\right)$, for $k=1, \ldots, n$, must be a linear combination of brackets that contain a single entry from $\left\{y, \alpha_{1}, \ldots, \alpha_{n}\right\}$ with all other entries the element $x$. Now $P\left(\alpha_{k}\right)$ is of odd degree and combining this fact with the previous remarks gives that each $P\left(\alpha_{k}\right)$ must be a linear combination of brackets which have one entry equal to $y$ and the rest equal to $x$. It follows that the perturbation $P$ must be of the form:

$$
\begin{aligned}
P\left(\alpha_{n}\right) & =\lambda_{n} \operatorname{ad}^{n+1}(x)(y) \\
& \vdots \\
P\left(\alpha_{k}\right) & =\lambda_{k} \operatorname{ad}^{k+1}(x)(y) \\
& \vdots \\
P\left(\alpha_{2}\right) & =\lambda_{2}[x,[x,[x, y]]] \\
P\left(\alpha_{1}\right) & =\lambda_{1}[x,[x, y]],
\end{aligned}
$$

with $P(x)$ and $P(y)$ both zero for degree reasons. Thus a perturbation $P$ can be specified by the $n$-tuple $\underline{\lambda}=\left(\lambda_{1}, \ldots, \lambda_{n}\right)$ with $\lambda_{k} \in \mathbb{Q}$, and such a perturbation is written $P_{\underline{\lambda}}$. Furthermore, any such $n$-tuple $\underline{\lambda}$ defines a perturbation since $d=0$ in the Quillen model of $H$, and hence $\left(d+P_{\underline{\lambda}}\right)^{2}=P_{\underline{\lambda}}^{2}=0$. In the Schlessinger-Stasheff terminology, all infinitesimal perturbations are unobstructed and hence are actually perturbations. Hence in this case, the variety $V_{H}$ is the $n$-dimensional vector space $\mathbb{Q}^{n}$. A typical point in $V_{H}$ is represented 
by an $n$-tuple $\underline{\lambda}$, corresponding to the perturbation

$$
P_{\underline{\lambda}}=\lambda_{1}[x,[x, y]] \cdot \partial \alpha_{1}+\cdots+\lambda_{n} \operatorname{ad}^{n+1}(x)(y) \cdot \partial \alpha_{n} .
$$

Now let $\underline{e}_{k}$ denote the $k$ th standard basis vector in $\mathbb{Q}^{n}$ for $k=1, \ldots, n$.

Claim. $V_{H} / \exp \left(\operatorname{ad} \mathscr{L}_{H}^{0}\right)=\left\{\left\{r \underline{e}_{k}\right\} \mid r \in \mathbb{Q}\right\}$.

Proof of Claim. The first step is to show that any perturbation $P_{\lambda}$ is equivalent to a perturbation of the form $P_{r \underline{e}_{k}}$, for some $r$ and $k$. If $\underline{\lambda}=(\overline{0}, \ldots, 0)$, this holds trivially, so let $\lambda_{m}$ be the first nonzero coordinate of $\underline{\lambda}$. If $m=n$, then $P_{\underline{\lambda}}=P_{\lambda_{n} \underline{\underline{e}}}$. If $1 \leq m<n$, then $P_{\underline{\lambda}} \sim_{\exp } P_{\lambda_{m} \underline{e}}$. To see this, consider the degree zero derivation $\theta$ defined by

$$
\begin{aligned}
\theta= & \left(\frac{\lambda_{m+1}}{\lambda_{m}}\right)\left[x, \alpha_{m}\right] \cdot \partial \alpha_{m+1}+\left(\frac{\lambda_{m+2}}{\lambda_{m}}\right)\left[x,\left[x, \alpha_{m}\right]\right] \cdot \partial \alpha_{m+2} \\
& +\cdots+\left(\frac{\lambda_{m+k}}{\lambda_{m}}\right) \operatorname{ad}^{k}(x)\left(\alpha_{m}\right) \cdot \partial \alpha_{m+k}+\cdots+\left(\frac{\lambda_{n}}{\lambda_{m}}\right) \operatorname{ad}^{n-m}(x)\left(\alpha_{m}\right) \cdot \partial \alpha_{n} .
\end{aligned}
$$

Now $\theta$ extends bracket length, so that $\theta \in \mathscr{L}_{H}^{0}$ and a straightforward check shows that

$$
\begin{aligned}
\exp (\operatorname{ad} \theta)\left(P_{\underline{\lambda}}\right) & =\left(P_{\underline{\lambda}}+\left[\theta, P_{\underline{\lambda}}\right]+\frac{1}{2 !}\left[\theta,\left[\theta, P_{\underline{\lambda}}\right]\right]+\cdots\right. \\
& =P_{\underline{\lambda}}-P_{\underline{\lambda}} \theta \\
& =P_{\underline{\lambda}}-\left(\lambda_{m} \operatorname{ad}^{m+1}(x)(y) \cdot \partial \alpha_{m}\right) \theta \\
& =\lambda_{m} \operatorname{ad}^{m+1}(x)(y) \cdot \partial \alpha_{m} \\
& =P_{\lambda_{m} \underline{e}_{m}} .
\end{aligned}
$$

It remains to show that two perturbations of the form $P_{r \underline{e}_{k}}$ and $P_{s \underline{e}_{l}}$ are not equivalent under the exponential action, unless $r \underline{e}_{k}=s \underline{e}_{l}$. But this follows since $P_{r \underline{e}_{k}}$ is a perturbation that extends bracket length homogeneously by $k+1$, so that for $\theta \in \mathscr{L}_{H}^{0}, \exp (\operatorname{ad} \theta)\left(P_{r \underline{e}_{k}}\right)$ can only differ from $P_{r \underline{e}_{k}}$ by terms that extend bracket length by at least $k+2$. This proves the claim.

We thus have a clear picture of the set of equivalence classes $V_{H} / \exp \left(\operatorname{ad} \mathscr{L}_{H}^{0}\right)$, in relation to the variety $V_{H}=\mathbb{Q}^{n}$. As a set, it can be pictured as the $n$ coordinate axes of $\mathbb{Q}^{n}$. To complete the classification of rational homotopy types that realize $H$, it remains to factor out by the action induced from Aut $H$. For this algebra $H$, the action is of a particularly simple form: The group Aut $H$ is isomorphic to the subgroup of $\mathrm{GL}(n+2, \mathbb{Q})$ consisting of diagonal matrices. Suppose that $\phi \in$ Aut $H$ induces the automorphism of $s^{-1} \widetilde{H}$ given by $\phi(x)=a x, \phi(y)=b y$, and $\phi\left(\alpha_{k}\right)=c_{k} \alpha_{k}$ for $k=1, \ldots, n$. Then the induced action on $V_{H} / \exp \left(\operatorname{ad} \mathscr{L}_{H}^{0}\right)$ is seen to be $\left\{P_{r \underline{e}_{k}}\right\} \mapsto\left\{P_{\text {se }_{k}}\right\}$, where $s=\left(a^{k+1} b / c_{k}\right) r$. Hence $\left\{P_{r \underline{e}_{k}}\right\} \sim_{\text {Aut }}\left\{P_{s \underline{e}_{k}}\right\}$ if $r s \neq 0$, for all $k=1, \ldots, n$. 
Thus the set $(*)$ has $n+1$ elements, $n$ of them corresponding to the nonzero axes in $\mathbb{Q}^{n}$ and the other one corresponding to the origin in $\mathbb{Q}^{n}$; and hence there are $n+1$ rational homotopy types that realize $H$.

Remark. It would be possible to prove Theorem 1 without referring to [SS]: One would establish directly the existence of a DG Lie algebra isomorphism between an arbitrary perturbation $\mathbb{L}\left(s^{-1} \widetilde{H}, P\right)$, and exactly one of $\mathbb{L}\left(s^{-1} \widetilde{H}\right)$ or the $n$ perturbations $\mathbb{L}\left(s^{-1} \widetilde{H}, P_{\underline{e}_{k}}\right)$. I use the ideas from [SS] because I feel their geometrical nature clarifies the proof. Here one has a good picture of the variety and the orbit spaces at each stage of the classification; this sequence of pictures helps one grasp what is required of the proof.

Similar examples for the dual question are now given. The proof is only sketched as it is analogous to the proof for the algebra $H$.

Theorem 2. Let $L=\pi_{*}(\Omega(K(\mathbb{Z}, 4) \times K(\mathbb{Z}, 4 n+1) \times X)) \otimes \mathbb{Q}$, where $X=$ $K(\mathbb{Z}, 4 n+8) \times K(\mathbb{Z}, 4 n+12) \times \cdots \times K(\mathbb{Z}, 8 n+4)$. Then $L$ is realized by exactly $n+1$ distinct rational homotopy types.

Sketch of proof. In this context, $V_{L} / \exp \left(\operatorname{ad} \mathscr{L}_{L}^{0}\right) /$ Aut $L$ is the appropriate classifying set. It is constructed in essentially the same way as the classifying set of Theorem 1, but starting from the Sullivan model of $L$ rather than the Quillen model. As before, the quotient $V_{L} / \exp \left(\operatorname{ad} \mathscr{L}_{L}^{0}\right)$ can be pictured as the $n$ coordinate axes in $V_{L}=\mathbb{Q}^{n}$, and an identical argument to that given in Theorem 1 shows that the classifying set has $n+1$ elements.

\section{CONCLUDING REMARKS}

A nice feature of these examples is that explicit representatives can be given for the $n+1$ rational homotopy types of Theorem 1. Each of these types corresponds to an orbit, under the two group actions, in the variety $V_{H}$. It is sufficient to choose a representative point from each orbit; the corresponding perturbation then gives a Quillen model for the rational homotopy type and from this model a space can be constructed. Referring to the proof of Theorem 1 , points in the variety that represent the $n+1$ orbits are the origin $\underline{0}$ and the $n$ standard basis vectors $\underline{e}_{1}, \ldots, \underline{e}_{n}$. These correspond to the perturbations 0 and $\operatorname{ad}^{k+1}(x)(y) \cdot \partial \alpha_{k}$ for $k=1, \ldots, n$. It follows that representatives of the $n+1$ rational homotopy types that realize $H$ can be taken as follows: $S^{5} \vee S^{4 n+4} \vee X$ as in the hypotheses of Theorem 1, together with the spaces

$$
S^{5} \vee S^{4 n+4} \vee \widehat{X}_{k} \cup_{f_{k}} e^{4 n+9+4 k} \text { for } k=1, \ldots, n,
$$

where $\widehat{X}_{k}=S^{4 n+13} \vee \cdots \vee S^{4 n+9+4(k-1)} \vee S^{4 n+9+4(k+1)} \vee \cdots \vee S^{8 n+9}$ and $f_{k}=$ $\operatorname{ad}^{k+1}\left(l_{5}\right)\left(l_{4 n+4}\right) \in \pi_{4 n+8+4 k}\left(S^{5} \vee S^{4 n+4}\right)$. Here, the 'ad' notation refers to the Whitehead product. Notice that $H$ is realized by $n+1$ rationally distinct spaces each of which is a finite complex and has Lusternik-Schnirelmann category $\leq 2$. In addition, each of these spaces is coformal [NM]. Indeed, it follows from 
[HL, Theorem 3.12] that the spaces have intrinsically coformal homotopy Lie algebras, i.e., each one is the unique realization of its homotopy Lie algebra. None of the spaces are formal, except the iterated wedge of spheres, which is both formal and coformal.

Now consider spaces that represent the $n+1$ rational homotopy types of Theorem 2. A description of these can be given that is analogous to the preceding discussion. Whereas the spaces for Theorem 1 are described in terms of cells and attaching maps, essentially using a homology decomposition; the spaces for Theorem 2 can be described as the total spaces of appropriate $K(\pi, n)$ fibrations, whose $k$-invariants are given by the corresponding perturbations and whose base spaces are products of Eilenberg-Mac Lane spaces. Details are left to the reader. In contrast to the spaces of Theorem 1, none of these are finite complexes and apart from the iterated product of Eilenberg-Mac Lane spaces, which is both coformal and formal, all are neither coformal nor formal. It would be interesting to know whether examples exist of Lie algebras realized by $n$ distinct rational homotopy types each represented by a finite complex, for any integer $n$.

\section{ACKNOWLEDGMENT}

I acknowledge, with pleasure, the substantial contribution of Ron Umble to this work. As a result of our collaboration, I developed something of a facility with the ideas contained in [SS], without which I do not think I could have constructed the examples of this paper.

\section{REFERENCES}

[BL] H. J. Baues and J. M. Lemaire, Minimal models in homotopy theory, Math. Ann. 225 (1977), 219-242.

[HL] S. Halperin and J.-M. Lemaire, Suites inertes dans les algèbres de Lie graduées, Math. Scand. 61 (1987), 39-67.

[LU] G. M. Lupton and R. N. Umble, Rational homotopy types with the rational cohomology of stunted complex projective space, preprint.

[NM] J. Neisendorfer and T. J. Miller, Formal and coformal spaces, Illinois J. Math. 22 (1978), 565-580.

[QU] D. Quillen, Rational homotopy theory, Ann. of Math. (2) 90 (1969), 205-295.

[SS] M. Schlessinger and J. Stasheff, Deformation theory and rational homotopy type, preprint.

Department of Mathematics and Computer Science, Dartmouth College, Hanover, NEW HAMPSHIRE 03755

Current address: Department of Mathematics, Cleveland State University, Cleveland, Ohio 44115 